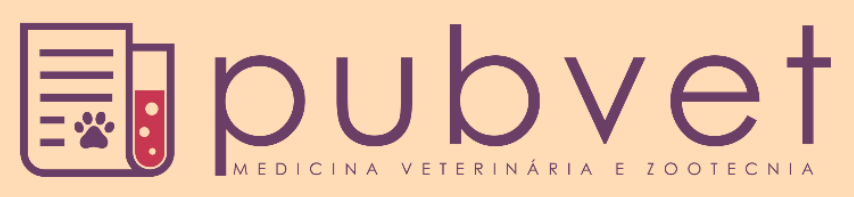

https://doi.org/10.31533/pubvet.v13n01a247.1-6

\title{
Comparação de técnicas coproparasitológicas no diagnóstico da coccidiose suína na baixada maranhense, Brasil
}

\author{
Thayane Ferreira Fernandes ${ }^{1 *}$, Leandro Henrique Veiga De Sousa ${ }^{1}{ }^{\bullet}$, Matheus Costa \\ Madeira $^{3}$, Fernando Moreira Vieira Souza ${ }^{3}$, Ana Clara Gomes Dos Santos ${ }^{2} \bullet$ \\ ${ }^{I}$ Mestrado em Ciência Animal, Departamento de Patologia, Universidade Estadual do Maranhão, São Luís-MA, Brasil \\ ${ }^{2}$ Departamento de Patologia, Universidade Estadual do Maranhão, São Luís-MA, Brasil. \\ ${ }^{3}$ Graduação em Medicina Veterinária, Departamento de Patologia, Universidade Estadual do Maranhão, São Luís-MA, Brasil \\ Autor para correspondência: thayane.ffer@gmail.com
}

\begin{abstract}
Resumo. O objetivo da pesquisa foi realizar a comparação de técnicas coproparasitológicas pelo método de Willis-Mollay (1921), Sheather (1923) e de sedimentação simples Hoffman et al. (1934), para determinar a melhor concentração de coccídios no diagnóstico da coccidiose em suínos da Baixada Maranhense, considerando o período seco e chuvoso. Foram analisadas 180 amostras fecais coletadas diretamente da ampola retal. Um total de 167 (92,7\%) suínos estavam parasitados. O método de Sheather apresentou maior eficácia na concentração dos oocistos. Dentre as técnicas utilizadas, a técnica de Sheather apresentou maiores concentrações de coccídeos para diagnóstico da coccidiose suína, no período seco exibindo maior carga parasitária.
\end{abstract}

Palavras chave: coccídeos, fezes, parasitas intestinais

\section{Comparison of coproparasitological techniques in the diagnosis of swine coccidiosis in the baixada maranhense, Brazil}

\begin{abstract}
The objective of this research was to compare coproparasitological techniques by Willis-Mollay (1921), Sheather (1923) and simple sedimentation method Hoffman et al. (1934) to determine the best concentration of coccidia in the diagnosis of coccidiosis in pigs of the Baixada Maranhense, considering the dry and rainy period. A total of 180 fecal samples were analyzed collected directly from the rectal ampulla. A total of 167 (92.7\%) pigs were parasitized. The Sheather method showed greater efficacy in oocyst concentration. Among the techniques used, the Sheather technique presented higher concentrations of coccidia for the diagnosis of swine coccidiosis, with the dry period exhibiting higher parasitism.
\end{abstract}

Keywords: coccidia, feces, intestinal parasites

\section{Comparación de técnicas coproparasitológicas en el diagnóstico de la coccidiosis porcina en la baixada maranhense, Brasil}

Resumen. El objetivo de la investigación fue realizar la comparación de técnicas coproparasitológicas por el método de Willis-Mollay (1921), Sheather (1923) e de sedimentación simples Hoffman et al. (1934), para determinar la mejor concentración de coccídios en el diagnóstico de la coccidiosis en porcinos de la Baixada Maranhense, considerando el período seco y lluvioso. Se analizaron 180 muestras fecales recogidas directamente de la ampolla rectal. Un total de 167 (92,7\%) cerdos estaban parasitados. El método de Sheather presentó una mayor eficacia en la concentración de los oocistos. Entre 
las técnicas utilizadas, la técnica de Sheather presentó mayores concentraciones de coccídeos para diagnóstico de la coccidiosis porcina, durante el período seco mostrando mayor carga parasitaria.

Palabras clave: coccídeos, heces, parásitos intestinales

\section{Introdução}

As doenças parasitárias são consideradas antigos problemas de saúde nas produções suinícolas, estando mais relacionadas às criações extensivas, comprometendo o desenvolvimento dos animais (Pinto et al., 2007). As características da coccidiose são influenciadas pelo tipo de produção implementado, fatores como a grande quantidade de animais facilita a disseminação da doença, deixando disponível no ambiente uma grande quantidade de oocistos (Lima, 2004).

A demonstração de oocistos nas fezes é útil para confirmar o diagnóstico de coccidiose (Schwartz, 2002). Com a finalidade de minimizar os resíduos fecais e facilitar a visualização de ovos, oocistos e larvas de helmintos gastrintestinais, são utilizados métodos parasitológicos qualitativos para a concentração dos oocistos de protozoários por meio de soluções hipersaturada de açúcar e Cloreto de Sódio $(\mathrm{NaCl})$ (Proudman \& Edwards, 1992). Os métodos de concentração são mais eficazes na eliminação de detritos fecais, extinguindo fragmentos que podem ser confundidas com oocistos, aumentando a possibilidade de encontrar o parasita, em animais com baixa carga parasitaria e assintomáticos (Huber et al., 2004).

O presente trabalho teve como objetivo realizar a comparação de técnicas coproparasitológicas pelo método de Willis-Mollay (1921), Sheather (1923) e de sedimentação simples de Hoffman et al. (1934) para determinar a melhor concentração de oocistos no diagnóstico de coccídeos, assim como foi realizado o método quantitativo de Gordon \& Whitlock (1939), para determinar o grau de parasitismo dos suínos da Baixada Maranhense, considerando o período seco e chuvoso.

\section{Material e métodos}

Esta pesquisa foi protocolada e aprovada sob o n ${ }^{\circ}$ 021/2014 no Comitê de Ética da Universidade Estadual do Maranhão.

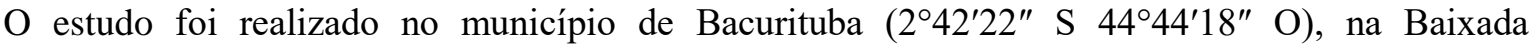
Maranhense, considerado como o polo de criação de suínos de forma rústica na região, sendo os animais criados a solta, no campo durante o período seco do ano e preso em retiros durante o período chuvoso sem separação de idade e sexo, sem manejo higiênico-sanitário e nutricional. A coleta foi realizada em seis criatórios, sendo três no período seco do ano (agosto a dezembro) e três no período chuvoso (janeiro a julho).

A escolha dos animais foi aleatória em cada criatório "chiqueiro" que apresentava um efetivo de 80 animais com idade entre 30 dias a 54 meses, de ambos os sexos. A coleta das fezes realizada na ampola retal em 30 animais, durante o período de abril de 2014 a setembro de 2015. Após a coleta, as fezes foram transportadas até o Laboratório de Parasitologia da Universidade Estadual do Maranhão. Para pesquisa de oocistos, foram utilizadas $4 \mathrm{~g}$ de fezes para cada método: Willis-Mollay (1921), Sheather (1923) e de sedimentação simples de Hoffman et al. (1934). Para o método quantitativo pela contagem de oocistos por grama de fezes (OoPG) foram utilizados $2 \mathrm{~g}$ de fezes, com uso da câmara de MacMaster, pelo método de Gordon \& Whitlock (1939) modificado, para avaliar a carga parasitária (CP) dos animais.

Os dados foram analisados pelo teste do Qui-quadrado considerando-se as comparações entre as técnicas coproparasitológicas e o período seco e chuvoso. Utilizou-se para isso a computação dos dados no Programa Excel for Windows, v. 2010, e análise estatística pelo SolfWare GraphPad Prism 500® (GraphPad Software, Inc., San Diego, CA, USA).

\section{Resultados e discussão}

Dos 180 suínos examinados, 92,7\% (167) estavam positivos para coccídeos, com maior frequência para as fêmeas adultas no período chuvoso e menor frequência para os machos adultos no período seco 
(Gráfico 1). Considerou-se nesse estudo como animais jovens até 12 meses de idade e adultos acima dessa faixa etária, de acordo com Mundim et al. (2004).

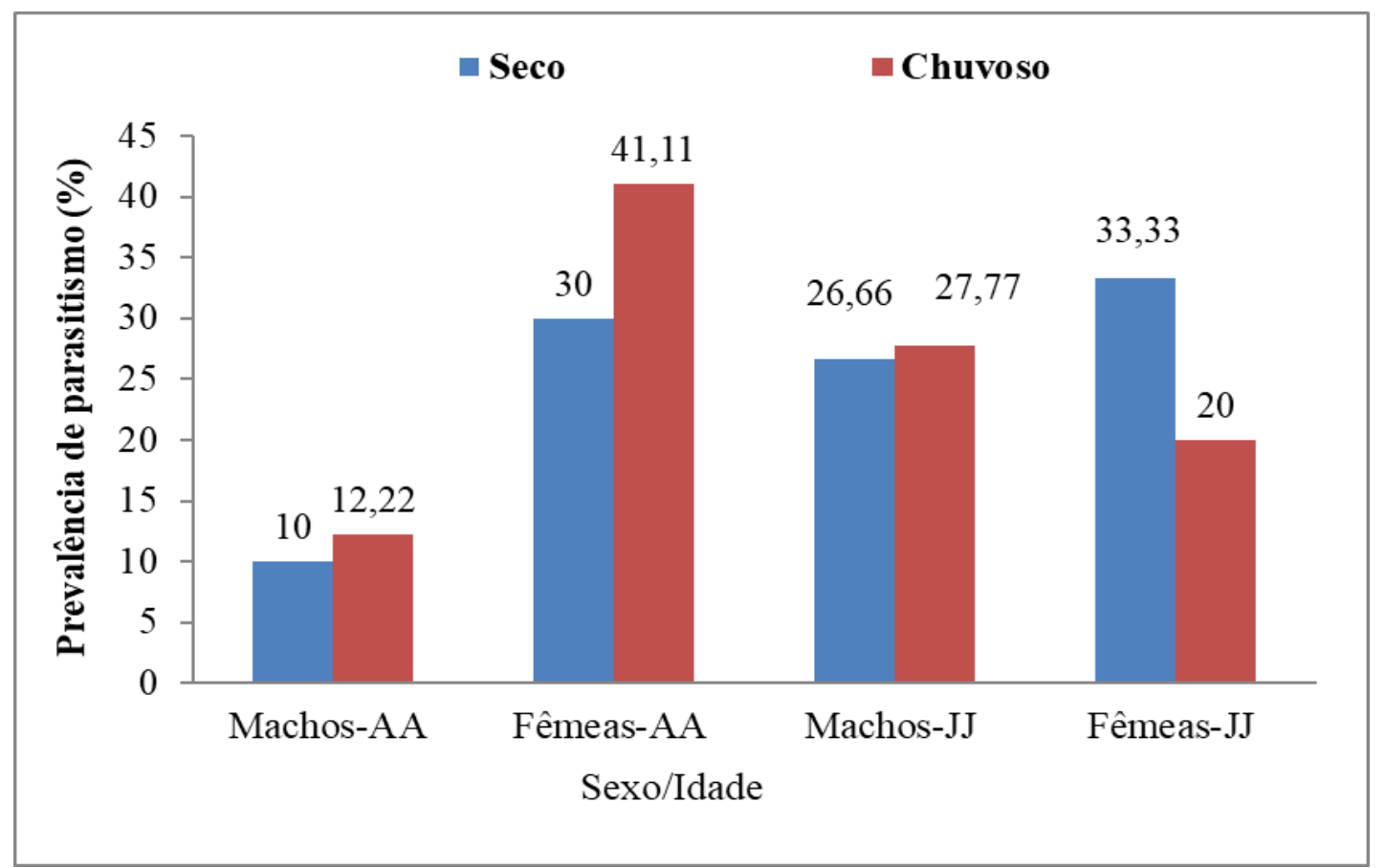

Gráfico 1. Frequência de parasitismo por coccídios intestinais em suínos da Baixada Maranhense, de acordo com o sexo, idade e período do ano, durante 2014 e 2015.

D’Alencar et al. (2010) encontraram baixa taxa de infecção nos suínos pesquisados em abatedouros da Região Metropolitana de Recife e da Zona da Mata do Estado de Pernambuco, que de acordo com os autores, pode ser explicada pelo fato das amostras terem sido oriundas de animais adultos. De acordo com Lima (2004), a coccidiose pode ocorrer em animais adultos, no entanto é basicamente uma infecção de animais jovens. Os casos de infecção em animais adultos estão relacionados com situações de alta densidade populacional, altas doses de oocistos eliminadas nas fezes, estresse, doenças oportunistas e deficiência imunitária.

Durante o período seco do ano na Baixada Maranhense, os campos permanecem secos, o solo com presença de torrões, formando-se pequeno lagos (as) e escassez de alimentos, com isso, os animais realizam grande caminhadas à procura de nutrientes, sendo desfavorável ao equilíbrio nutricional e fisiológico, propiciando a exacerbação do parasitismo, tanto por nematódeos como protozoários intestinais. Durante o período chuvoso esses animais ficam confinados em "retiros" e recebem alimentação no cocho; além do gasto energético ser menor, por não fazerem longas caminhadas, retendo assim mais energia.

Dentre os métodos coproparasitológicos realizados, o método de Sheather (1923) apresentou maior frequência na concentração de coccídeos, nas três coletas realizadas em cada período do ano, seco e chuvoso. Obteve-se uma variação de 67,2 a $96,1 \%$ para o período seco, e 75,9 a $93,6 \%$ para o período chuvoso. O método de Willis-Mollay (1921) apresentou a menor concentração de coccídeos, quando comparado aos demais, demonstrando que o método de Sheather (1923) de Hoffman et al. (1934) para os suínos são mais eficazes para pesquisas desses parasitos (Gráfico 2). No período seco foi verificado um parasitismo por 23900; 23100 e 41700 OoPG. O período chuvoso apresentou 20200; 19100 e 15700 OoPG, comprovando sua validação, pela contagem de oocistos.

$\mathrm{Na}$ análise dos resultados, o teste do Qui-Quadrado, demonstrou que durante o período seco e chuvoso, a concentração do número de coccídeos para os métodos coproparasitológicos realizados apresentou diferença estatística significativa, dentre eles $(\mathrm{P}<0,0001)$, ressaltando que o método de Sheather foi o que concentrou maior número de oocistos, seguido ao de Hoffman et al. (1934), que não apresentou diferença estatística significativa, entre ambos $(P>0,07)$, no entanto o método de Wilis- 
Mollay apresentou menor concentração de oocistos, diferindo dos demais métodos anteriormente citados, isto é, com diferença estatística significativa $(\mathrm{P}<0,001)$ (Tabela 1).

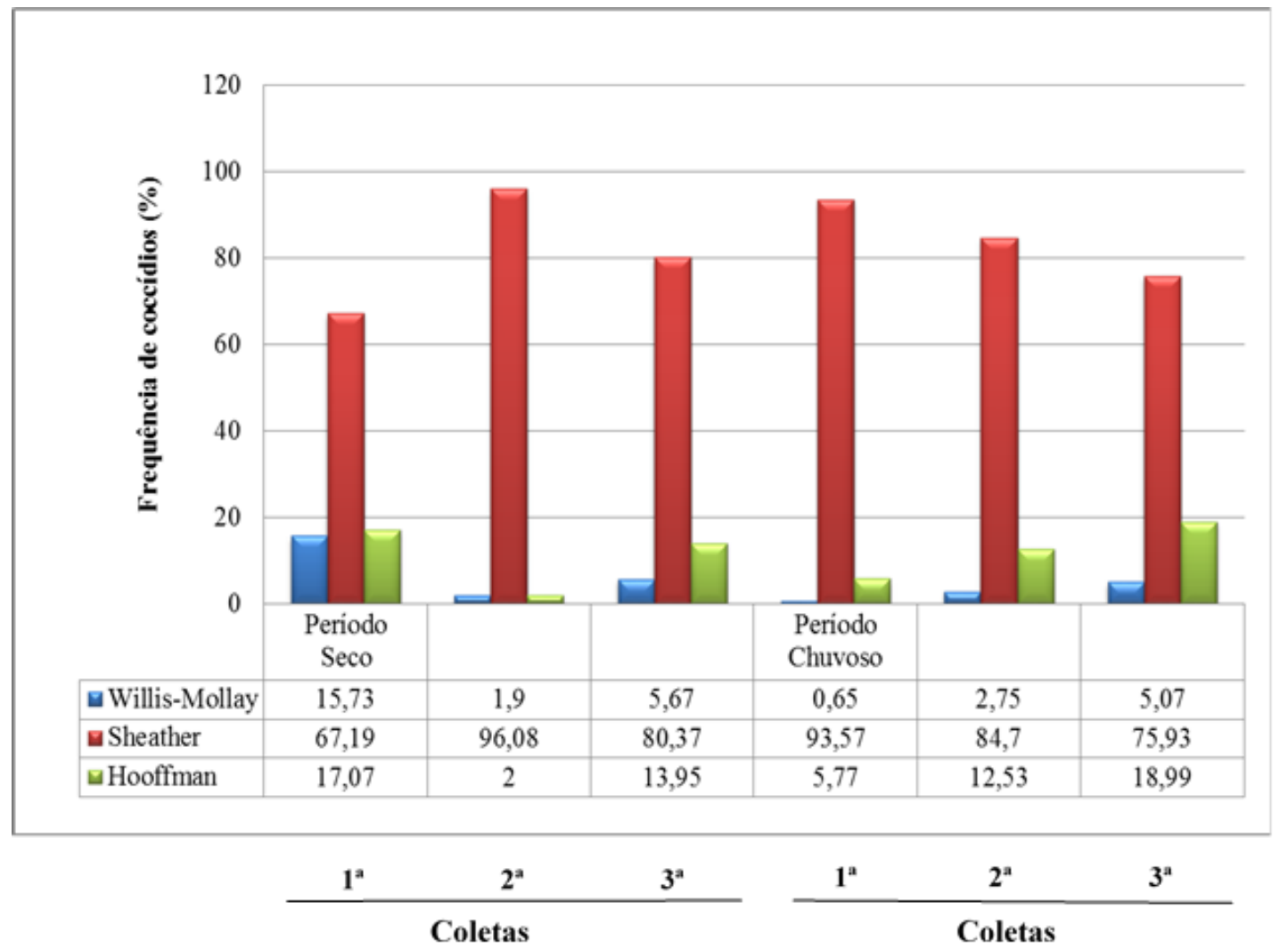

Gráfico 2. Frequência de coccídeos de suínos da Baixada Maranhense, entre métodos coproparasitológicos, no período seco e chuvoso durante 2014 a 2015.

Tabela 1. Comparação do número coccídeos intestinais de suínos da Baixada Maranhense, quantificado pelos métodos coproparasitológicos, durante o período seco e chuvoso, de 2014 a 2015.

\begin{tabular}{lcccc}
\hline \multirow{2}{*}{ Período do ano } & \multicolumn{3}{c}{ Métodos coproparasitológicos } & \multirow{2}{*}{ Total } \\
\cline { 2 - 4 } & Willis-Mollay & Sheather & Hooffman & \\
\hline Seco & N. $(\%)$ & $20231(83,0)$ bd & $2713(11,13) \mathrm{cd}$ & 24373 \\
Chuvoso & $1429(5,86) \mathrm{a}$ & $12297(86,73)$ bd & $1552(10,94) \mathrm{cd}$ & 14177 \\
\hline Total & $328(2,31) \mathrm{a}$ & $32528(84,37)$ & $4265(11,06)$ & 38550 \\
\hline
\end{tabular}

Letras minúsculas iguais na horizontal não diferem entre si pelo Teste do Qui-Quadrado e teste de Tukey $(\mathrm{P}<0,0001)$

Como já demonstrado, a técnica de Sheather (1923), detectou maior concentração de coccídeos, por ser uma técnica mais sensível e especifica. De acordo com Hoff et al. (2005), o uso da centrifugação aumenta a aderência de oocistos na lâmina, fato este que explica a diferença encontrada entre as técnicas pesquisadas. Na detecção de oocistos de protozoários, as técnicas de centrifugação são determinantes para o diagnóstico (Huber et al., 2003).

O método de centrífugo-flutuação em solução hipersaturada de açúcar normalmente é o mais comum utilizado na detecção de oocistos em amostras fecais (Current, 1990). De acordo com Xiao \& Feng (2008), após serem processadas, as amostras precisam ser analisadas evitando exposições prolongadas a soluções com alta densidade, para evitar alterações na morfologia dos oocistos, acarretando resultados equivocados.

O método de flutuação é insuficiente para diagnóstico quando os oocistos não são eliminados durante a fase aguda da doença, podendo ser encontrados após a essa fase. No entanto, mesmo parasitado, o animal pode não liberar oocistos nas fezes quando a recolha das amostras incidirem entre os picos de 
eliminação (Hoffmann, 1987; Radostits et al., 2010). O método de flutuação em solução de Sheather é mais comumente usado na identificação de coccídeos em animais por aumentarem as chances de encontrar oocistos, especialmente em animais assintomáticos. Contudo, existem outras soluções de flutuação que também podem ser empregadas, como Sulfato de Zinco. As técnicas de sedimentação por Formalina-Éter ou Acetato de Etila são utilizadas para exames em que o material fecal apresenta uma grande quantidade de gordura (Lindsay et al., 1997). Já, as técnicas de sedimentação são amplamente utilizadas para concentrar cistos de protozoários, como são técnicas que se baseiam num processo de lavagem, não concentram tanto oocistos como nas técnicas de flutuação.

Geralmente os suínos de criação intensiva apresentam uma fauna parasitária abaixo daqueles de criação extensiva (Roepstorff \& Jorsal, 1990), estes apresentam parasitismo tanto em animais jovens quanto em adultos, mesmo assim, os sistemas extensivos com manejo adequado não exibem desvantagens quando relacionados ao sistema intensivo (White, 1996).

O aumento da criação suinícola extensiva fez reaparecer determinados parasitas já controlados no meio intensivo, o controle parasitário nessas criações se torna um desafio aos agricultores e profissionais da saúde animal (Fortes, 2004). O histórico do animal e a presença de sinais clínicos precisam ser analisados para o diagnóstico definitivo, sendo que a presença em si dos oocistos nas fezes não justifica a confirmação do mesmo, visto que os oocistos podem ser localizados em animais perfeitamente saudáveis (Bowman, 2004; Bowman, 2014).

Por este trabalho, observou-se que no estudo comparativo entre as técnicas coproparasitológicas no diagnóstico de coccidioses intestinais de suínos, a técnica de Sheather apresenta melhor diagnóstico ao parasitismo, devido a maior concentração de oocistos do gênero Eimeria e Cystoisospora.

\section{Agradecimentos}

A Fundação de Amparo à Pesquisa e ao Desenvolvimento Científico e Tecnológico do Maranhão FAPEMA.

\section{Referências bibliográficas}

Bowman D. (2004). Parasitologia veterinária de Georgis. Elsevier Brasil.

Bowman D.D. (2014). Georgis' Parasitology for Veterinarians-E-Book. Saunders Company, USA.

Current W.L. (1990). Techniques and laboratory maintenance of Cryptosporidium. In: Cryptosporidiosis of man and animals (eds. by Dubey JP, Speer CA \& Fayer R), pp. 59-82. Boca Raton Press, São Pauoo, Brasil.

D’Alencar A.S., Farias M.P.O., Rosas M.M., Lima M.M., Alves L.C. \& Faustino M., A. G. (2010). Monitoria patológica e inquérito epidemiológico para avaliação da infecção por helmintos e coccídios em suínos de abatedouros da Região Metropolitana de Recife e Zona da Mata do estado de Pernambuco. Ciência Veterinária nos Trópicos 13, 52-59.

Fortes E. (2004). Parasitologia veterinária. Editora ícone, São Paulo.

Gordon H.M. \& Whitlock H.V. (1939). A new technique for counting nematode eggs in sheep faeces. Journal of the council for Scientific and Industrial Research 12, 50-52.

Hoff G., Silva A.S. \& Monteiro S.G. (2005). Avaliação do parasitismo e comparação de técnicas de análise fecal em suínos de granjas da região oeste do estado de Santa Catarina. Revista da FZVA 12, 106-115.

Hoffman W.A., Pons J.A. \& Janer J.L. (1934). The sedimentation-concentration method in Schistosomiasis mansoni. Journal Public Health 9, 283-298.

Hoffmann R.P. (1987) Diagnóstico de parasitismo veterinário. Sulina, Porto Alegre, Rio Grande do Sul, Brasil.

Huber F., Bomfim T.C. \& Gomes R.S. (2003). Comparação da eficiência da técnica de Sedimentação pelo formaldeído-éter e da técnica de centrífugo-flutuação modificada na detecção de cistos de Giardia sp. e oocistos de Cryptosporidium sp. em amostras fecais de bezerros. Revista Brasileira de Parasitologia Veterinária 12, 135-137. 
Huber F., Bomfim T.C. \& Gomes R.S. (2004). Comparação da eficiência da coloração pelo método da safranina a quente e da técnica de centrífugo-flutuação na detecção de oocistos de Cryptosporidium em amostras fecais de animais domésticos. Revista Brasileira de Parasitologia Veterinária 13, 8184.

Lima J.D. (2004) Coccidiose dos ruminantes domésticos. Revista Brasileira de Parasitologia Veterinária 13, 9-13.

Lindsay D.S., Dubey J.P. \& Blagburn B.L. (1997). Biology of Isospora spp. from humans, nonhuman primates, and domestic animals. Clinical Microbiology Reviews 10, 19-34.

Mundim M.J.S., Mundim A.V., Santos A.L.Q., Cabral D.D., Faria E.S.M. \& Moraes F.M. (2004). Helmintos e protozoários em fezes de javalis (Sus scrofa scrofa) criados em cativeiro Helminths and protozoa in wild boars (Sus scrofa scrofa) feces raised in captivity. Arquivo Brasileiro de Medicina Veterinária e Zootecnia 56, 792-795.

Pinto J.M.S., Costa J.O. \& Souza J.C.A. (2007). Ocorrência de endoparasitos em suínos criados em Itabuna, Bahia, Brasil. Ciência Veterinária nos Trópicos 10, 79-85.

Proudman C.J. \& Edwards G.B. (1992). Validation of a centrifugation/flotation technique for the diagnosis of equine cestodiasis. The Veterinary Record 131, 71-72.

Radostits O.M., Gay C.C., Blood D.C., Hinchcliff K.W. \& McKenzie R.A. (2010). Clínica Veterinária: um tratado de doenças dos bovinos, ovinos, suínos, caprinos e eqüinos. Guanabara Koogan, Rio de Janeiro.

Roepstorff A. \& Jorsal S.E. (1990). Relationship of the prevalence of swine helminths to management practices and anthelmintic treatment in Danish sow herds. Veterinary Parasitology 36, 245-257.

Schwartz, K. (2002). Swine Coccidiosis. Cooperative Extension Service, Michigan State University.

Sheather A.L. (1923). The Detection of Intestinal Protozoa and Mange Parasites by a Floatation Technique. Journal of Pathology and Therapy 36, 266-275.

White M. (1996). Control in the outdoors. Pigs 3, 28-30.

Willis-Mollay H.H. (1921). A simple levitation method for the detection of hookworm ova. Medical Journal of Australia 2, 375-376.

Xiao L. \& Feng Y. (2008). Zoonotic cryptosporidiosis. FEMS Immunology \& Medical Microbiology 52, 309-323.

Recebido: 9 dezembro, 2018

Aprovado: 29 dezembro, 2018.

Publicado: 14 janeiro, 2019.

Licenciamento: Este artigo é publicado na modalidade Acesso Aberto sob a licença Creative Commons Atribuição 4.0 (CC-BY 4.0), a qual permite uso irrestrito, distribuição, reprodução em qualquer meio, desde que o autor e a fonte sejam devidamente creditados. 